Research Article

\title{
Deterministic Sudden Changes and Stochastic Fluctuation Effects on Stability and Persistence Dynamics of Two-Predator One-Prey Model
}

\author{
Jawdat Alebraheem $\mathbb{D}^{1},{ }^{1}$ Nasser S. Elazab, ${ }^{2}$ Mogtaba Mohammed, ${ }^{1}$ Anis Riahi, ${ }^{1}$ \\ and Ahmed Elmoasry \\ ${ }^{1}$ Department of Mathematics, College of Science Al Zufli, Majmaah University, Majmaah 11952, Saudi Arabia \\ ${ }^{2}$ Department of Mathematics, Faculty of Science, Cairo University, Giza, Egypt
}

Correspondence should be addressed to Jawdat Alebraheem; j.alebraheem@mu.edu.sa

Received 26 November 2020; Revised 14 January 2021; Accepted 1 February 2021; Published 3 March 2021

Academic Editor: Hijaz Ahmad

Copyright (c) 2021 Jawdat Alebraheem et al. This is an open access article distributed under the Creative Commons Attribution License, which permits unrestricted use, distribution, and reproduction in any medium, provided the original work is properly cited.

\begin{abstract}
In this paper, we present new results on deterministic sudden changes and stochastic fluctuations' effects on the dynamics of a two-predator one-prey model. We purpose to study the dynamics of the model with some impacting factors as the problem statement. The methodology depends on investigating the seasonality and stochastic terms which make the predator-prey interactions more realistic. A theoretical analysis is introduced for studying the effects of sudden deterministic changes, using three different cases of sudden changes. We show that the system in a good situation presents persistence dynamics only as a stable dynamical behavior. However, the system in a bad situation leads to three main outcomes as follows: first, constancy at the initial conditions of the prey and predators; second, extinction of the whole system; third, extinction of both predators, resulting in the growth of the prey population until it reaches a peak carrying capacity. We perform numerical simulations to study effects of stochastic fluctuations, which show that noise strength leads to an increase in the oscillations in the dynamical behavior and became more complex and finally leads to extinction when the strength of the noise is high. The random noises transfer the dynamical behavior from the equilibrium case to the oscillation case, which describes some unstable environments.
\end{abstract}

\section{Introduction}

1.1. Preface. Theoretical ecology has motivated many mathematicians to discuss different ideas and models from a purely mathematical standpoint; see for example [1-5]. Mathematical modeling is a useful tool to determine how a process works and to predict what may follow [3]. Many problems taken into consideration in mathematical ecology seem simple, but are considered complicated problems due to the difficulty of determining the underlying ecological principles [6]. Nonlinear differential equations are used to mathematically describe predator-prey interactions. However, it is typically difficult to find a suitable mathematical analysis, especially when using nonlinear terms.
The Lotka-Volterra model, which is a system of nonlinear coupled first-order ordinary differential equations, has been deemed the basic model for describing predator-prey interactions $[7,8]$. Two-predator one-prey models have the form of three species interactions, and thus, these systems are described by a system of three equations. Their dynamical behavior has been studied by some researchers [9-11].

Seasonality is an important factor, which plays a vital role in describing the changes and fluctuations in ecological systems with predator-prey interactions [12-18]. Additionally, there are many ecological factors, such as hunting and climate, which have varied effects (positive and/or negative) on the dynamical behaviors of the species. In the literature, a number of studies have investigated the effect of 
seasonality on the dynamical behavior of predator-prey systems, but most of these studies have focused on the search of chaotic cases in predator-prey systems [12-14]. Several researchers $[13,15]$ have used impulsive differential equations to describe steep changes, where they studied the systems over a long period. However, in this paper, we will use the novel tool over describing steep changes for a long time or as a new situation is introduced into the system.

Deterministic models have been widely used to describe predator-prey interactions and their dynamics. Deterministic models are useful, due to their ability to follow them through mathematical analysis, and they are an important mechanism for describing stable environments. However, random fluctuations appear in unstable environments, so deterministic models are difficult to describe these environments. In addition, the random noises are an important tool to conclude some unexpected dynamical behaviors of predator-prey interactions. Stochastic models play an important role for describing more realistic dynamical modeling of ecosystems. May [19] introduced an important contribution when he investigated stochastic differential equations for describing the limits of niche overlap in a randomly fluctuating environment. Recently, stochastic predator-prey models and their dynamics have been studied by some researchers [20-25].

The study of the dynamical behavior of predator-prey interactions has been considered to be an important subject in applied mathematics and mathematical ecology, due to its universal existence and importance [26]. Stability is one of the main important dynamics of predator-prey systems, which is typically the first property considered when studying dynamical behavior.

The persistence and extinction dynamics have also been discussed by many researchers [27-30], due to their importance. The analytical definitions of persistence and extinction are as follows: for a population $p(t)$, if $p(0)>0$ and $\lim _{t \rightarrow \infty} p(t)>0$, then $p(t)$ persists, while if $p(0)>0$ and $\lim _{t \rightarrow \infty} p(t)=0$, then $p(t)$ becomes extinct. The geometric meaning of persistence is defined that each trajectory of a system of differential equations is bounded away from the coordinate axes, but the geometric meaning of extinction is that the trajectory of the system of differential equations touches the coordinate axes.

The novelty of our work is on consideration of the deterministic and stochastic models taken in such a way; we are to get several results through our analysis. It should be noted that, we transfer the nonautonomous model to the autonomous model(s) by using a novel tool that approximates the model to particular cases.

In this paper, we aim to investigate a cosinusoidal function in a Holling type I two-predator one-prey model, in order to study how sudden changes of the dynamics will effect on the dynamical behavior of the model. Investigating the cosinusoidal function and stochastic terms make our assumptions more realistic by concluding new cases of the model. We transfer the nonautonomous model to the autonomous model(s) by using a novel tool which approximates the model to particular cases.

The paper is arranged as follows: we introduce in Section 1, the preface and methodology of the paper. In Section 2, we present the mathematical model of the two-predator oneprey system and the seasonality function. In Section 3, we introduce forced deterministic models by sudden changes, divided to two situations: bad and good. In Section 4, we present a mathematical analysis of the deterministic sudden changes. In Section 5, we study the equilibrium points and conduct a stability analysis of these situations. In Section 6, we introduce the stochastic model of the two-predator oneprey system and the numerical simulations. In Section 7, we summarize our conclusions.

1.2. The Methodology. I summarize the mechanism that is followed in this paper through Figure 1.

The methodology of arrays:

Array 1: adding the stochastic term

Array 2: adding the seasonality function

Array 3: using the approximation method

Array 4: theoretical analysis

Array 5: numerical simulation

\section{Mathematical Model and Seasonality Function}

2.1. Mathematical Model. We use a nondimensional system of the Holling type I two-predator one-prey model [31] as follows:

$$
\begin{aligned}
& \frac{\mathrm{d} x}{\mathrm{~d} t}=x\left(1-\frac{x}{k}\right)-\alpha x y-\beta x z, \\
& \frac{\mathrm{d} y}{\mathrm{~d} t}=-u y+e_{1} \alpha x y-e_{1} \alpha y^{2}-c_{1} y z, \\
& \frac{\mathrm{d} z}{\mathrm{~d} t}=-u z+e_{2} \beta x z-e_{2} \beta z^{2}-c_{2} y z,
\end{aligned}
$$

subject to initial conditions

$$
\begin{aligned}
& x(0)=x_{0}>0, \\
& y(0)=y_{0}>0, \\
& z(0)=z_{0}>0 .
\end{aligned}
$$

The biological meaning of the variables and parameters is as follows:

$$
\begin{aligned}
& x \text { : prey density } \\
& y \text { : first predator density } \\
& z \text { : second predator density } \\
& k \text { : carrying capacity of the system } \\
& \alpha \text { and } \beta \text { : searching and capturing efficiency of predators } \\
& y \text { and } z \\
& u \text { and } w \text { : loss rates of predators } y \text { and } z \\
& e_{1} \text { and } e_{2} \text { : birth rate of the predator for each prey } \\
& \text { consumed } \\
& c_{1} \text { and } c_{2} \text { : interspecific competition between the } \\
& \text { predators }
\end{aligned}
$$




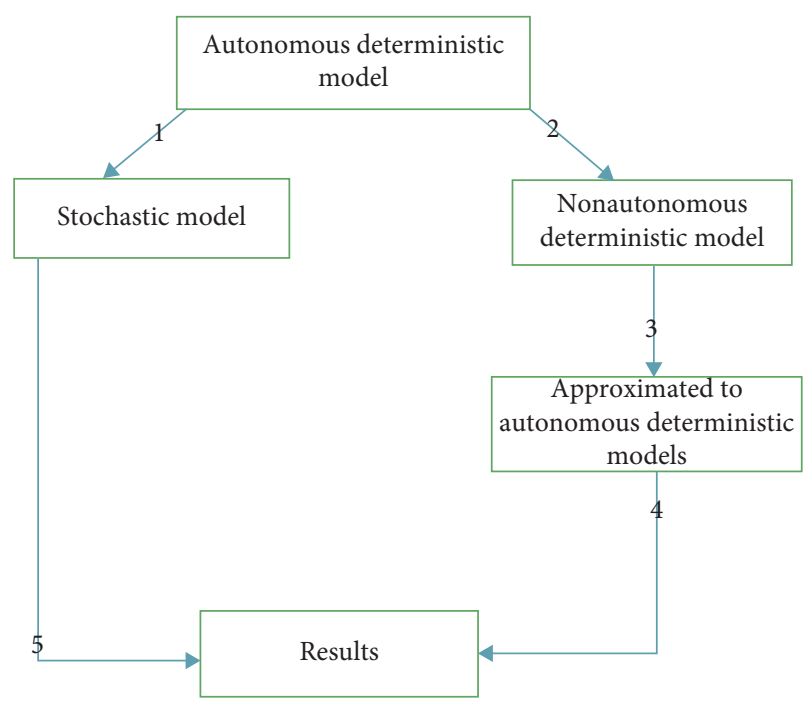

Figure 1: Summary of methodology.

The parameters and initial conditions of the model (1) are supposed to be positive values.

Theorem 1. All the solutions of system (1) which initiate in $R_{+}^{3}$ for $t \geq 0$ are bounded.

Proof. according to the first equation of system (1), we prove that it is bounded as follows:

$$
\frac{\mathrm{d} x}{\mathrm{~d} t} \leq x\left(1-\frac{x}{k}\right)
$$

The solution of equation (3) is $x(t)=k e^{t+k c} /\left(-1+e^{t+k c}\right)$, where $c$ is the integration constant.

Then, $0 \leq \lim _{t \rightarrow \infty} \sup x(t) \leq k \forall t>0$.

Then, we prove that $x(t)+y(t)+z(t) \leq Q, \forall t \geq 0$.

Let $R(t)=x(t)+y(t)+z(t)$.

The derivative of $R$ with respect to time $(t)$ is as follows:

$$
\begin{aligned}
\frac{\mathrm{d} R}{\mathrm{~d} t}= & \frac{\mathrm{d} x}{\mathrm{~d} t}+\frac{\mathrm{d} y}{\mathrm{~d} t}+\frac{\mathrm{d} z}{\mathrm{~d} t} \\
\frac{\mathrm{d} R}{\mathrm{~d} t}= & \left(\left(1-\frac{x}{k}\right)-\alpha y-\beta z\right) x+\left(-u+e_{1} \alpha x-e_{1} \alpha y-c_{1} z\right) y \\
& +\left(-u+e_{2} \beta x-e_{2} \beta z-c_{2} y\right) z .
\end{aligned}
$$

Since all the parameters are positive and the solutions initiating continue in the nonnegative quadrant in $R_{+}^{3}$, we can suppose the following:

$$
\frac{\mathrm{d} R}{\mathrm{~d} t}=\left(1-\frac{x}{k}\right) x+\left(-u+e_{1} \alpha x-e_{1} \alpha y\right) y+\left(-u+e_{2} \beta x-e_{2} \beta z\right) z .
$$

We have that

$$
\max \left\{R_{+} x\left(1-\frac{x}{k}\right)\right\}=\frac{k}{4}
$$

By substituting in (5), it becomes as follows:

$$
\begin{aligned}
& \frac{\mathrm{d} R}{\mathrm{~d} t} \leq \frac{k}{4}+\left(-u+e_{1} \alpha x-e_{1} \alpha y\right) y+\left(-w+e_{2} \beta x-e_{2} \beta z\right) z \\
& \frac{\mathrm{d} R}{\mathrm{~d} t} \leq \frac{k}{4}+\left(-u+e_{1} \alpha x-e_{1} \alpha y\right) y+\left(-w+e_{2} \beta x-e_{2} \beta z\right) z \\
&+R(t)-R(t) .
\end{aligned}
$$

Equation (8) can be written as follows:

$$
\begin{aligned}
\frac{\mathrm{d} R}{\mathrm{~d} t}+R(t) \leq & \frac{K}{4}+x+\left(-u+e_{1} \alpha x-e_{1} \alpha y+1\right) y \\
& +\left(-w+e_{2} \beta x-e_{2} \beta z+1\right) z .
\end{aligned}
$$

Since $x(t) \leq k$, then

$$
\begin{aligned}
\frac{\mathrm{d} R}{\mathrm{~d} t}+R(t) \leq & \frac{5 k}{4}+\left(-u+e_{1} \alpha-e_{1} \alpha y+1\right) y \\
& +\left(-w+e_{2} \beta x-e_{2} \beta z+1\right) z
\end{aligned}
$$

but

$$
\begin{aligned}
& \max _{R_{+}}\left\{\left(-u+e_{1} \alpha k-e_{1} \alpha y+1\right) y\right\}=\frac{-1+e_{1} \alpha k^{2}-2 e_{1} \alpha u k+u^{2}}{4 e_{1} \alpha}, \\
& \max _{R_{+}}\left\{\left(-w+e_{2} \beta k-e_{2} \beta z+1\right) z\right\}=\frac{-1+e_{2} \beta k^{2}-2 e_{2} \beta w k+w^{2}}{4 e_{2} \beta} .
\end{aligned}
$$

So, equation (10) becomes as follows:

$$
\frac{\mathrm{d} R}{\mathrm{~d} t}+R(t) \leq Q,
$$

where

$$
Q=\frac{1}{4}\left(5 k+\frac{-1+e_{1} \alpha k^{2}-2 e_{1} \alpha u k+u^{2}}{e_{1} \alpha}+\frac{-1+e_{2} \beta k^{2}-2 e_{2} \beta w k+w^{2}}{e_{2} \beta}\right) \text {. }
$$

Thus, $R(t) \leq Q+\rho e^{-t}$, where $\rho$ is a constant of integration:

$$
\underset{t \rightarrow \infty}{\limsup } R(t) \leq \lim _{t \rightarrow \infty} Q+\rho e^{-t} .
$$

Then, $R(t) \leq Q$.

2.2. Seasonality Function. Cosinusoidal and sinusoidal functions $[12,14,16]$ are used for describing the effects of seasonality on the dynamical behavior of the model (1). The cosinusoidal function is as follows:

$$
C(t)=1+\epsilon \cos (\mu t)
$$

where the parameter $\epsilon$ indicates the seasonality degree (or strength seasonal degree) and the parameter $\mu$ represents the angular frequency of the fluctuations caused by impacts. 


\section{Forced Deterministic Models by Sudden Changes}

Events that happen unexpectedly (i.e., as the result of some environmental factors) on predator-prey interactions are called sudden changes. We apply the approximation method to describe such changes, in order to simplify the mathematical analysis of the model and make it biologically sensible. The approximation method has been applied for analyzing SIR models by some researchers [31, 32]. However, Alebraheem [16] has applied this technique to transfer a nonautonomous model containing seasonality terms to a autonomous model(s) by approximating the model to particular cases, in order to study the dynamical behavior of predator-prey systems.

We apply the approximation method by taking the smallest and biggest values of the seasonality degree $\epsilon$, where $0 \leq \epsilon \leq 1$. Hence, we approximate the cosinusoidal function (equation (15)) by the following two situations:

$$
C(t) \cong P(t)= \begin{cases}0, & \text { bad situation, } \\ 2, & \text { good situation. }\end{cases}
$$

We interpret the "bad" and "good" situations as indicating surrounding circumstances are bad or good, respectively.

We investigate the cosinusoidal function (equation (15)) in system (3) through three different cases, as follows.

If sudden changes are forced for the whole system, we have the following:

$$
\begin{aligned}
& \frac{\mathrm{d} x}{\mathrm{~d} t}=\left(x\left(1-\frac{x}{k}\right)-\alpha x y-\beta x z\right) P(t), \\
& \frac{\mathrm{d} y}{\mathrm{~d} t}=\left(-u y+e_{1} \alpha x y-e_{1} \alpha y^{2}-c_{1} y z\right) P(t), \\
& \frac{\mathrm{d} z}{\mathrm{~d} t}=\left(-w z+e_{2} \beta x z-e_{2} \beta z^{2}-c_{2} y z\right) P(t) .
\end{aligned}
$$

If sudden changes are forced for the prey species through the growth rate of the prey, we have the following:

$$
\begin{aligned}
& \frac{\mathrm{d} x}{\mathrm{~d} t}=P(t) x\left(1-\frac{x}{k}\right)-\alpha x y-\beta x z, \\
& \frac{\mathrm{d} y}{\mathrm{~d} t}=-u y+e_{1} \alpha x y-e_{1} \alpha y^{2}-c_{1} y z, \\
& \frac{\mathrm{d} z}{\mathrm{~d} t}=-w z+e_{2} \beta x z-e_{2} \beta z^{2}-c_{2} y z .
\end{aligned}
$$

If sudden changes are forced for both predator' species through the birth rate of the predator for each prey consumed, we have the following:

$$
\begin{aligned}
& \frac{\mathrm{d} x}{\mathrm{~d} t}=x\left(1-\frac{x}{k}\right)-\alpha x y-\beta x z, \\
& \frac{\mathrm{d} y}{\mathrm{~d} t}=-u y+e_{1} P(t) \alpha x y-e_{1} \alpha y^{2}-c_{1} y z, \\
& \frac{\mathrm{d} z}{\mathrm{~d} t}=-w z+e_{2} P(t) \beta x z-e_{2} \beta P(t) z^{2}-c_{2} y z .
\end{aligned}
$$

\section{Mathematical Analysis of Deterministic Sudden Changes}

In this section, we analyze the sudden changes' effects on system (3) mathematically, so we substitute the values of $P(t)$ (equation (16)) through three cases (i.e., systems (17)-(19)) as follows:

The first case: if sudden changes have an effect on the whole system.

The bad situation: when we use $P(t)=0$, system (17) becomes as follows:

$$
\begin{aligned}
& \frac{\mathrm{d} x}{\mathrm{~d} t}=0, \\
& \frac{\mathrm{d} y}{\mathrm{~d} t}=0, \\
& \frac{\mathrm{d} z}{\mathrm{~d} t}=0 .
\end{aligned}
$$

The solutions of equations (20a)-(20c) are as follows:

$$
\begin{aligned}
& \lim _{t \longrightarrow \infty} x(t)=x_{0}, \\
& \lim _{t \longrightarrow \infty} y(t)=y_{0}, \\
& \lim _{t \longrightarrow \infty} z(t)=z_{0} .
\end{aligned}
$$

We conclude from systems (20a)-(20c), the system will be set at the initial conditions.

The good situation: when we use $P(t)=2$, system (17) becomes as follows:

$$
\begin{aligned}
& \frac{\mathrm{d} x}{\mathrm{~d} t}=2 x\left(1-\frac{x}{k}\right)-2 \alpha x y-2 \beta x z, \\
& \frac{\mathrm{d} y}{\mathrm{~d} t}=-2 u y+2 e_{1} \alpha x y-2 e_{1} \alpha y^{2}-2 c_{1} y z, \\
& \frac{\mathrm{d} z}{\mathrm{~d} t}=-2 w z+2 e_{2} \beta x z-2 e_{2} \beta z^{2}-2 c_{2} y z .
\end{aligned}
$$

The second case: if sudden changes have an effect on the prey species through the growth rate of the prey.

The bad situation: when we use $P(t)=0$, system (18) becomes as follows:

$$
\begin{aligned}
& \frac{\mathrm{d} x}{\mathrm{~d} t}=(0-\alpha x y-\beta x z), \\
& \frac{\mathrm{d} y}{\mathrm{~d} t}=\left(-u y+e_{1} \alpha x y-e_{1} \alpha y^{2}-c_{1} y z\right), \\
& \frac{\mathrm{d} z}{\mathrm{~d} t}=\left(-w z+e_{2} \beta x z-e_{2} \beta z^{2}-c_{2} y z\right) .
\end{aligned}
$$

The solution of equation (23a) becomes as follows.

Because $y>0$ and $z>0$, so we can reduce equation (23a) to become the following: 


$$
\begin{array}{r}
\frac{\mathrm{d} x}{\mathrm{~d} t}=-\alpha x, \\
x(t)=e^{-\alpha t},
\end{array}
$$

then $\lim _{t \rightarrow \infty} x(t)=0$.

Since $y$ and $z$ follow $x$, then $\lim _{t \rightarrow \infty} y(t)=0$ and $\lim _{t \rightarrow \infty} z(t)=0$.

The good situation: when we use $P(t)=2$, system (18) becomes as follows:

$$
\begin{aligned}
& \frac{\mathrm{d} x}{\mathrm{~d} t}=2 x\left(1-\frac{x}{k}\right)-\alpha x y-\beta x z, \\
& \frac{\mathrm{d} y}{\mathrm{~d} t}=-u y+e_{1} \alpha x y-e_{1} \alpha x y^{2}-c_{1} y z, \\
& \frac{\mathrm{d} z}{\mathrm{~d} t}=-w z+e_{2} \beta x z-e_{2} \beta S(t) z^{2}-c_{2} y .
\end{aligned}
$$

The third case: if sudden changes have an effect on both predator' species through the birth rate of the predator for each prey consumed.

The bad situation: when we use $P(t)=0$, system (19) becomes as follows:

$$
\begin{aligned}
& \frac{\mathrm{d} x}{\mathrm{~d} t}=x\left(1-\frac{x}{k}\right)-\alpha x y-\beta x z, \\
& \frac{\mathrm{d} y}{\mathrm{~d} t}=-u y+0-0-c_{1} y z, \\
& \frac{\mathrm{d} z}{\mathrm{~d} t}=-w z+0-0-c_{2} y z .
\end{aligned}
$$

For equations (26b) and (26c), we remove the terms $-c_{1} y z$ and $-c_{2} y z$ because they are negative terms and to simplify the mathematical analysis, so we have the following:

$$
\begin{aligned}
& \frac{\mathrm{d} y}{\mathrm{~d} t}=-u y-c_{1} y z \cong-u y, \\
& \frac{\mathrm{d} z}{\mathrm{~d} t}=-w z-c_{2} y z \cong-w z .
\end{aligned}
$$

The solution of equation (27) is as follows:

$$
y(t)=y_{0} e^{-u t} \text {. }
$$

Then, the solution leads to the following:

$$
\lim _{t \longrightarrow \infty} y(t)=0 \text {. }
$$

The solution of equation (28) is as follows:

$$
z(t)=z_{0} e^{-u t}
$$

The solution of this equation leads to the following:

$$
\lim _{t \rightarrow \infty} z(t)=0
$$

Since $\lim _{t \rightarrow \infty} y(t)=0$ and $\lim _{t \rightarrow \infty} z(t)=0$, then equation (26a) becomes as follows:

$$
\frac{\mathrm{d} x}{\mathrm{~d} t}=x\left(1-\frac{x}{k}\right)
$$

The solution of equation (26a) is as follows:

$$
x(t)=\frac{k e^{t+k c}}{-1+e^{t+k c}},
$$

where $c$ is the integration constant, then $\lim _{t \rightarrow \infty} x(t)=k$.

The good situation: when we use $P(t)=2$, system (19) becomes as follows:

$$
\begin{aligned}
& \frac{\mathrm{d} x}{\mathrm{~d} t}=x\left(1-\frac{x}{k}\right)-\alpha x y-\beta x z \\
& \frac{\mathrm{d} y}{\mathrm{~d} t}=-u y+2 e_{1} \alpha x y-2 e_{1} \alpha y^{2}-c_{1} y z \\
& \frac{\mathrm{d} z}{\mathrm{~d} t}=-w z+2 e_{2} \beta x z-2 e_{2} \beta z^{2}-c_{2} y z
\end{aligned}
$$

\section{Equilibrium Points and Stability Analysis}

One of the main dynamical behaviors is stability. We find the positive equilibrium points to study the stability. To check the local stability, we compute the variational matrices corresponding to each equilibrium point and using the Routh-Hurwitz criterion for studying the stability. To check the global stability, we do that by constructing the Dulac function and Lyapunov function and using them to prove the global stability. We summarize the results of the equilibrium points of good situations when the sudden changes are forced through three cases in the following table (Table 1).

We present only the proof of the first case, and in the same manner, the proofs of the second and third cases will be followed, so the proofs of the second and third cases will be omitted.

\section{Theorem 2}

(i) The trivial equilibrium point $E_{0}=(0,0,0)$ is a saddle point

(ii) The peak equilibrium point $E_{1}=(k, 0,0)$ is locally asymptotically stable in $x$-direction, but it is locally asymptotically stable in $y-z$ plane if it holds the conditions (38) and (39)

Proof

(i) we compute the variational matrix of $E_{0}$ which is given as follows:

$$
M_{1}=\left(\begin{array}{ccc}
2 & 0 & 0 \\
0 & -2 u & 0 \\
0 & 0 & -2 w
\end{array}\right) \text {. }
$$

Through the variational matrix $M_{1}$, we see that the eigenvalues of $y$-direction and $z$-direction are negative, but the 


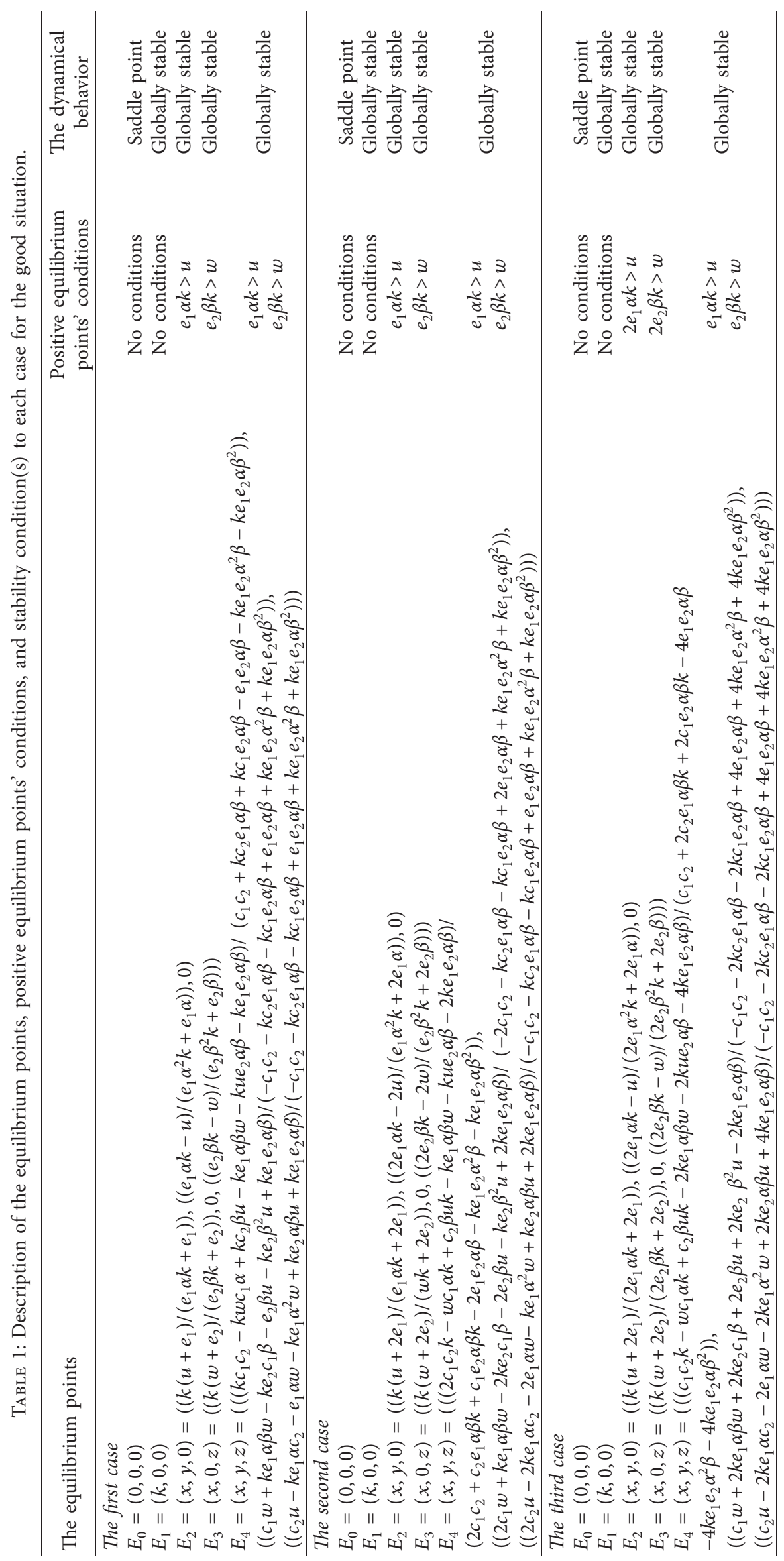


eigenvalue of $x$-direction is positive; this explains that the manifold is unstable along $x$-direction, but stable along $y$ direction and along $z$-direction. Then, the trivial equilibrium point $E_{0}$ is the saddle point.

(ii) The variational matrix of $E_{1}$ is given as follows:

$$
M_{2}=\left(\begin{array}{ccc}
-2 & -2 k \alpha & -2 k \beta \\
0 & -2 u+2 e_{1} \alpha k & 0 \\
0 & 0 & -2 w+2 e_{2} \beta k
\end{array}\right) .
$$

Through the variational matrix $M_{2}$, we notice that the equilibrium point $E_{1}$ is locally asymptotically stable, if the following conditions are satisfied:

$$
\begin{aligned}
& u>e_{1} \alpha k, \\
& w>e_{2} \beta k .
\end{aligned}
$$

Theorem 3. The peak equilibrium point $E_{1}=(k, 0,0)$ is globally asymptotically stable under the following conditions:

$$
\begin{aligned}
& u>4 e_{1} \alpha^{2} k, \\
& w>4 e_{2} \beta^{2} k .
\end{aligned}
$$

Proof. Consider the following Lyapunov function about $E_{1}$ :

$$
V_{1}=\left(x-k-k \ln \left(\frac{x}{k}\right)\right)+\frac{y}{2 e_{1} \alpha}+\frac{z}{2 e_{2} \beta} \text {. }
$$

where $V_{1}$ is a continuously differentiable real-valued function defined on $R_{+}^{3}$. Therefore, we have the following:

$$
\begin{aligned}
\frac{\mathrm{d} V_{1}}{\mathrm{~d} t}= & \left(1-\frac{\bar{x}}{x}\right) \frac{\mathrm{d} x}{\mathrm{~d} t}+\frac{1}{2 e_{1} \alpha} \frac{\mathrm{d} y}{\mathrm{~d} t}+\frac{1}{2 e_{2} \beta} \frac{\mathrm{d} z}{\mathrm{~d} t}, \\
\frac{\mathrm{d} V_{1}}{\mathrm{~d} t}= & B_{1} \frac{(x-k)}{x} x\left[1-\frac{x}{k}-2 \alpha y-2 \beta z\right] \\
& +\frac{y}{2 e_{1} \alpha}\left[-u+2 e_{1} \alpha x-2 e_{1} \alpha y-c_{1} z\right] \\
& +\frac{z}{2 e_{2} \beta}\left[-w+2 e_{2} \beta x-2 e_{2} \beta z-c_{2} y\right], \\
\frac{\mathrm{d} V_{1}}{\mathrm{~d} t}= & -\frac{1}{k}(x-k)^{2}-y^{2}-z^{2}-2 \alpha x y-2 \beta x z \\
& -\left(\frac{2 e_{2} \beta c_{1}+2 e_{1} \alpha c_{2}}{4 e_{1} e_{2} \alpha \beta}\right) y z-\left(\frac{u-4 e_{1} \alpha^{2} k}{2 e_{1} \alpha}\right) y \\
& -\left(\frac{w-4 e_{2} \beta^{2} k}{2 e_{2} \beta}\right) z .
\end{aligned}
$$

If the conditions (40) and (41) are satisfied, then we obtain that $\left(\mathrm{d} V_{1} / \mathrm{d} t\right)<0$ for any point in $R_{+}^{3}$.

\section{Theorem 4}

(i) The equilibrium point $E_{2}$ is globally asymptotically stable in the interior of the positive quadrant of $x-y$ plane

(ii) The equilibrium point $E_{3}$ is globally asymptotically stable in the interior of the positive quadrant of $x-z$ plane

We prove part (i), and in the same manner, part (ii) can be proved.

Proof. let $G(x, y)=1 / x y$, where $G$ is a Dulac function. It is continuously differentiable in the positive quadrant of the $x-y$ plane $A=\{(x, y) \mid x>0, y>0\}$ :

$$
\begin{aligned}
& N_{1}(x, y)=2 x\left(1-\frac{x}{k}\right)-2 \alpha x y, \\
& N_{2}(x, y)=-2 u y+2 e_{1} \alpha x y-2 e_{1} \alpha y^{2} .
\end{aligned}
$$

Thus, $\Delta\left(\mathrm{GN}_{1}, \mathrm{GN}_{2}\right)=\left(\partial\left(\mathrm{GN}_{1}\right) / \partial x\right)+\left(\partial\left(\mathrm{GN}_{2}\right) / \partial y\right)=$ $(-2 / y k)-\left(2 e_{1} \alpha / x\right)$.

We find that $\Delta\left(\mathrm{GN}_{1}, \mathrm{GN}_{2}\right)<0$ for all $x>0$ and $y>0$ in the positive quadrant of the $x-y$ plane. By using Bendixson-Dulac criterion, there is no periodic solution in the interior of the positive quadrant of the $x-y$ plane. $E_{2}$ is globally asymptotically stable in the interior of the positive quadrant of the $x-y$ plane.

Theorem 5. The persistence equilibrium point $\widehat{E}=(\hat{x}, \hat{y}, \widehat{z})$ of system (22) is globally asymptotically stable.

Proof: we use the Lyapunov function to prove the global stability of positive equilibrium point $\widehat{E}$ as follows:

$$
\begin{aligned}
V= & B_{1}\left(x-\hat{x}-\hat{x} \ln \left(\frac{x}{\overline{\hat{x}}}\right)\right)+B_{2}\left(y-\hat{y}-\hat{y} \ln \left(\frac{y}{\widehat{y}}\right)\right) \\
& +B_{3}\left(z-\widehat{z}-\widehat{z} \ln \left(\frac{z}{\widehat{z}}\right)\right) .
\end{aligned}
$$

Equation (45) can be expressed as follows:

$$
V=B_{1} h_{1}(x, \bar{x})+B_{2} h_{2}(y, \bar{y})+B_{3} h_{3}(z, \bar{z}),
$$

where $h_{1}(x, \bar{x})=x-\bar{x}-\bar{x} \ln (x / \bar{x})$

$$
\begin{aligned}
& h_{2}(y, \bar{y})=y-\bar{y}-\bar{y} \ln \left(\frac{y}{\bar{y}}\right), \\
& h_{3}(z, \bar{z})=z-\bar{z}-\bar{z} \ln \left(\frac{z}{\bar{z}}\right) .
\end{aligned}
$$

System (22) can be written as follows: 


$$
\begin{aligned}
& \frac{\mathrm{d} x}{\mathrm{~d} t}=x J(x, y, z), \\
& \frac{\mathrm{d} y}{\mathrm{~d} t}=y L_{1}(x, y, z), \\
& \frac{\mathrm{d} z}{\mathrm{~d} t}=z L_{2}(x, y, z),
\end{aligned}
$$

where

$$
\begin{gathered}
J(x, y, z)=2-2 \frac{x}{k}-2 \alpha y-2 \beta z, \\
L_{1}(x, y, z)=-2 u+2 e_{1} \alpha x-2 e_{1} \alpha y-2 c_{1} z, \\
L_{2}(x, y, z)=-2 w+2 e_{2} \beta x-2 e_{2} \beta z-2 c_{2} y .
\end{gathered}
$$

Let

$$
h^{\prime}(\lambda, \bar{\lambda})=\frac{\partial h}{\partial \lambda}(\lambda, \bar{\lambda})=1-\frac{\bar{\lambda}}{\lambda}
$$

We compute the derivative of $V$ along the trajectories of system (22):

$$
\frac{\mathrm{d} V}{\mathrm{~d} t}=B_{1} h_{1}^{\prime}(x, \bar{x}) \frac{\mathrm{d} x}{\mathrm{~d} t}+B_{2} h_{2}^{\prime}(y, \bar{y}) \frac{\mathrm{d} y}{\mathrm{~d} t}+B_{3} h_{3}^{\prime}(z, \bar{z}) \frac{\mathrm{d} z}{\mathrm{~d} t},
$$

which is

$$
\begin{aligned}
\frac{\mathrm{d} V}{\mathrm{~d} t}=B_{1}\left(1-\frac{\bar{x}}{x}\right) \frac{\mathrm{d} x}{\mathrm{~d} t}+B_{2}\left(1-\frac{\bar{y}}{y}\right) \frac{\mathrm{d} y}{\mathrm{~d} t}+B_{3}\left(1-\frac{\bar{z}}{z}\right) \frac{\mathrm{d} z}{\mathrm{~d} t} & \\
\frac{\mathrm{d} V}{\mathrm{~d} t}= & B_{1} \frac{(x-\bar{x})}{x} x[J(x, y, z)]+B_{2} \frac{(y-\bar{y})}{y} y\left[L_{1}(x, y, z)\right] \\
& +B_{3} \frac{(z-\bar{z})}{z} z\left[L_{2}(x, y, z)\right] .
\end{aligned}
$$

Equation (53) can be expressed as follows:

$$
\begin{aligned}
= & B_{1} \frac{(x-\bar{x})}{x} x[J(x, y, z)-J(\bar{x}, \bar{y}, \bar{z})] \\
& +B_{2} \frac{(y-\bar{y})}{y} y\left[L_{1}(x, y, z)-L_{1}(\bar{x}, \bar{y}, \bar{z})\right] \\
& +B_{3} \frac{(z-\bar{z})}{z} z\left[L_{2}(x, y, z)-L_{2}(\bar{x}, \bar{y}, \bar{z})\right] .
\end{aligned}
$$

where $J(\bar{x}, \bar{y}, \bar{z})=0, L_{1}(\bar{x}, \bar{y}, \bar{z})=0$, and $L_{2}(\bar{x}, \bar{y}, \bar{z})=0$, so we have

$$
\begin{aligned}
\frac{\mathrm{d} V}{\mathrm{~d} t}= & B_{1}(x-\hat{x})\left[\left[2-2 \frac{x}{k}-2 \alpha y-2 \beta z\right]\right. \\
& \left.-\left[2-2 \frac{\widehat{x}}{k}-2 \alpha \widehat{y}-2 \beta \widehat{z}\right]\right]+B_{2}(y-\widehat{y}) \\
& \cdot\left[\left[-2 u+2 e_{1} \alpha x-2 e_{1} \alpha y-2 c_{1} z\right]\right. \\
& \left.-\left[-2 u+2 e_{1} \alpha \widehat{x}-2 e_{1} \alpha \widehat{y}-2 c_{1} \widehat{z}\right]\right] \\
& +B_{3}(z-\widehat{z})\left[\left[-2 w+2 e_{2} \beta x-2 e_{2} \beta z-2 c_{2} y\right]\right. \\
& \left.-\left[-2 w+2 e_{2} \beta \widehat{x}-2 e_{2} \beta \widehat{z}-2 c_{2} \hat{y}\right]\right] .
\end{aligned}
$$

Rearrange the terms of equation (55):

$$
\begin{aligned}
\frac{\mathrm{d} V}{\mathrm{~d} t}= & \frac{B_{1}}{k}(x-\widehat{x})[-2(x-\widehat{x})-2 \alpha(y-\widehat{y})-2 \beta(z-\widehat{z})]+B_{2}(y-\widehat{y})\left[2 e_{1} \alpha(x-\widehat{x})-2 e_{1} \alpha(y-\bar{y})-2 c_{1}(z-\widehat{z})\right] \\
& +B_{3}(z-\widehat{z})\left[2 e_{2} \beta(x-\widehat{x})-2 e_{2} \beta(z-\widehat{z})-2 c_{2}(y-\widehat{y})\right] \\
\frac{\mathrm{d} V}{\mathrm{~d} t}= & \frac{B_{1}}{k}\left[-2(x-\widehat{x})^{2}-2 \alpha(x-\widehat{x})(y-\widehat{y})-2 \beta(x-\widehat{x})(z-\widehat{z})\right] \\
& +B_{2}\left[2 e_{1} \alpha(y-\widehat{y})(x-\widehat{x})-2 e_{1} \alpha(y-\widehat{y})^{2}-2 c_{1}(y-\widehat{y})(z-\widehat{z})\right] \\
& +B_{3}\left[2 e_{2} \beta(z-\widehat{z})(x-\widehat{x})-2 e_{2} \beta(z-\widehat{z})^{2}-2 c_{2}(z-\widehat{z})(y-\widehat{y})\right] .
\end{aligned}
$$


By selecting $B_{1}=1, B_{2}=1 / e_{1}$, and $B_{3}=1 / e_{2}$, so

$$
\begin{aligned}
\frac{\mathrm{d} V}{\mathrm{~d} t}= & -\frac{2}{k}(x-\widehat{x})^{2}-2 \alpha(y-\widehat{y})^{2}-2 \frac{c_{1}}{e_{1}}(y-\widehat{y})(z-\widehat{z}) \\
& -2 \beta(z-\widehat{z})^{2}-2 \frac{c_{2}}{e_{2}}(z-\widehat{z})(y-\widehat{y}) .
\end{aligned}
$$

We find that $\mathrm{d} V / \mathrm{d} t$ is negative under no condition (i.e., no restrictions on parameters).

From Theorem 5, we notice that the persistence dynamical behaviors of system (22) are globally stable.

\section{Stochastic Model}

In this section, we give numerical simulation to the stochastic version of our model. This consideration is due to the prevalence of randomness in almost all wild animal life, which makes the use of stochastic differential equations more realistic and efficient to describe some predictions of dynamical behaviors, see Figures 2-5.

The standard Itô stochastic differential equation is written as follows [24]:

$$
\begin{aligned}
& \mathrm{d} x(t)=F(t, x(t)) \mathrm{d} t+G(t, x(t)) \mathrm{d} W(t), \\
& x\left(t_{0}\right)=x_{0},
\end{aligned}
$$

where the first term represents the drift coefficient and the second term represents the random noise in the environment, which is sometimes called Gaussian white noise.

We use a stochastic term in the deterministic model (2) as in reference [24], so we have the following model:

$$
\begin{aligned}
& \frac{\mathrm{d} x}{\mathrm{~d} t}=x\left(1-\frac{x}{k}\right)-\alpha x y-\beta x z+\sigma_{1} x \mathrm{~d} W_{1}, \\
& \frac{\mathrm{d} y}{\mathrm{~d} t}=-u y+e_{1} \alpha x y-e_{1} \alpha y^{2}-c_{1} y z+\sigma_{2} y \mathrm{~d} W_{2}, \\
& \frac{\mathrm{d} z}{\mathrm{~d} t}=-w z+e_{2} \beta x z-e_{2} \beta z^{2}-c_{2} y z+\sigma_{3} z \mathrm{~d} W_{3},
\end{aligned}
$$

where $\sigma_{i}, i=1,2,3$ represent the strength of noise, and $\mathrm{d} W_{i}$, $i=1,2,3$ is a standard Wiener or Brownian motion processes.

We have theoretically proven that the dynamical behavior of deterministic sudden changes is globally stable. In this section, we present the effects' stochastic fluctuations on the dynamical behavior. The MATHEMATICA program was used to perform the numerical simulations. The values of parameters were selected to fulfil the positive values of a nontrivial equilibrium point, called the coexistence point (i.e., to satisfy conditions (38) and (39)) in deterministic models. In addition, the values of $\sigma_{i}=\sigma$ were set as in [24], to represent three levels of noise strength; that is, low, medium, and high noise strengths. The parameters and initial conditions' values were taken as follows:

$$
k=2.0, \alpha=1.0, \beta=1.4, e_{1}=0.6, e_{2}=0.65, c_{1}=0.07,
$$
$c_{2}=0.04, u=0.45, w=0.6, x(0)=0.6, \quad y(0)=0.3$, and $z(0)=0.25$

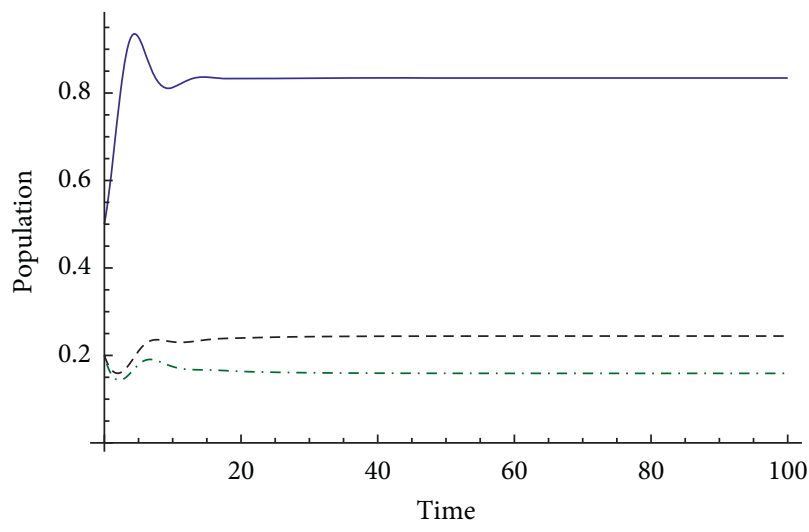

$$
\begin{aligned}
& -x(t) \\
& ---y(t) \\
& -.-z(t)
\end{aligned}
$$

Figure 2: Time series of system (59) without noise $(\sigma=0.0)$.

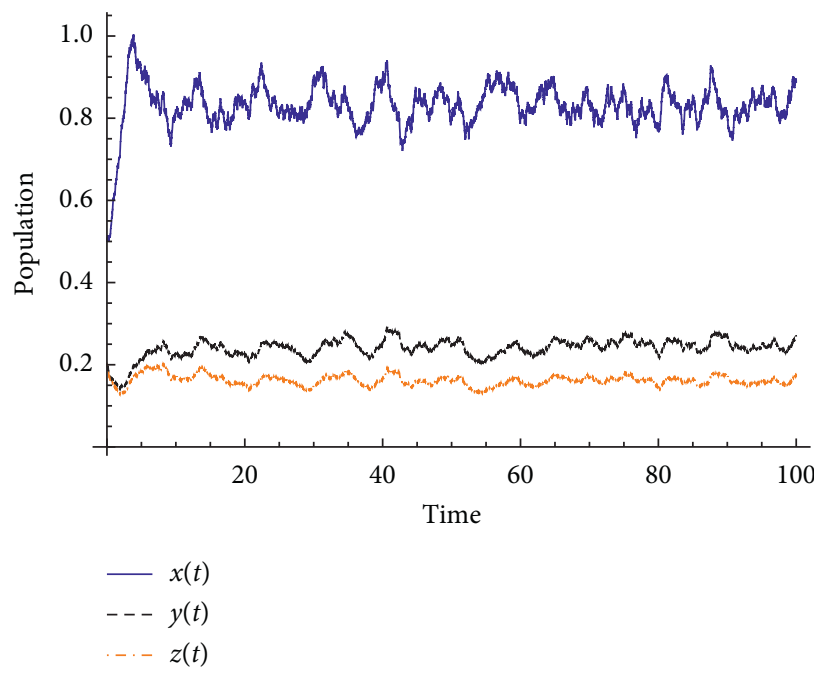

FIgURE 3: Time series of system (59) with low noise strength $(\sigma=0.05)$.

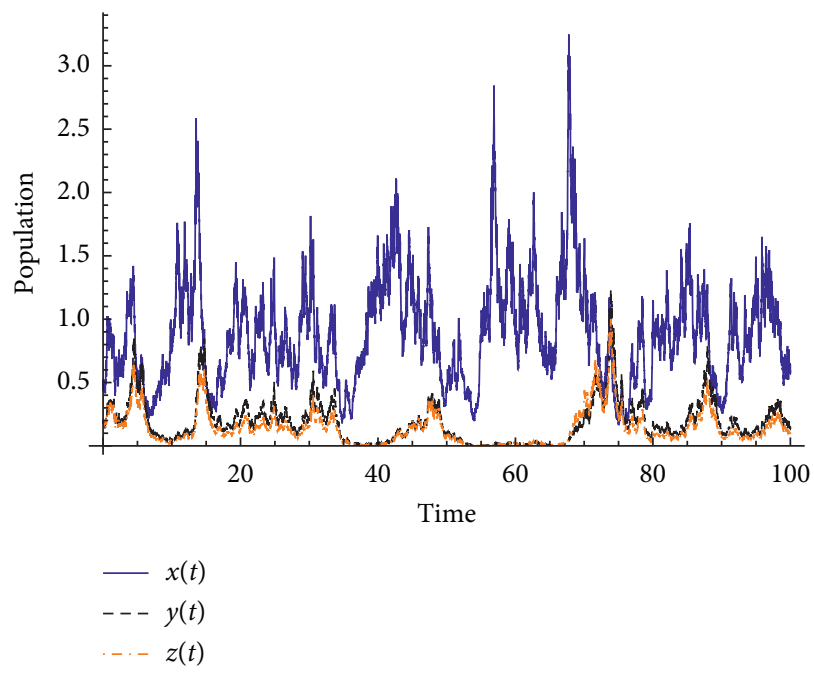

FIgURE 4: Time series of system (59) with medium noise strength $(\sigma=0.5)$. 


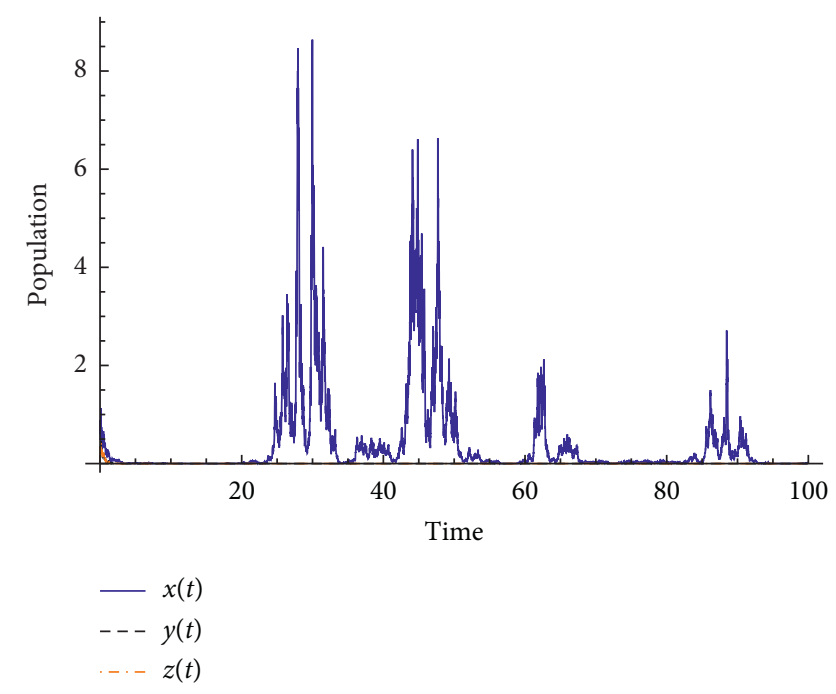

Figure 5: Time series of system (59) with high noise strength $(\sigma=1.4)$.

Figure 2 represents the dynamical behavior of the model (59) without noise (i.e., $\sigma=0$ ), which gives the deterministic model. In Figure 2, the dynamical behavior of the species was stable coexistence, which corresponds with the theoretical analysis of the deterministic model. Figure 3 represents the dynamical behavior of the model (59) when the strength of the noise was low. Figure 3 shows that the dynamical behavior of the species was coexistence with smooth oscillations. However, with an increase in the strength of noise, such as the mediumnoise situation shown in Figure 4, the dynamical behavior of the species was coexistence with sharp oscillations, which may lead to extinction. Figure 5 represents the dynamical behavior of the model (59) when the noise strength was high. The dynamical behavior of the species became more complex, and they tended to extinction. The random noises transfer the dynamical behavior from the equilibrium case to the oscillation case, which describes some unstable environments.

We conclude that increasing the noise strength led to an increase of oscillation in the dynamical behavior, which can be interpreted biologically as increasing the probability of extinction, representing the worst-case scenario of dynamical behavior. This result corresponds with the numerical simulations. These results correspond well with the results of reference [24] with the difference being the mathematical model used, whereas increasing the noise strength led to an increase in oscillations in the dynamical behavior, finally leading to extinction when the noise strength was high.

\section{Conclusion}

We investigated the seasonality effects in a Holling type I two-predator one-prey model, which can more realistically describe the species of interaction more realistic. The nonautonomous models are transferred to autonomous models by approximating the model to particular cases representing sudden changes, so the situations are classified to bad and good situations, according to the surrounding circumstances. A mathematical analysis of sudden changes is introduced, and the equilibrium points and stability are discussed. We made the following conclusions.

For the bad situations, we obtained the following outcomes:

If sudden fluctuations have an effect on the whole system, then the system will remain at the initial conditions

If sudden fluctuations have an effect on the prey species, then both predators' species and the prey species will go extinct

If sudden fluctuations have an effect on both predator' species, then the prey species will reach carrying capacity, while both of the predator' species will go extinct The equilibrium points of each case were obtained and found to be stable

For the good situations, we obtained the following outcomes:

The one-prey two-predators system interacted through three different systems (22), (25), and (35) which represented the three cases

We obtained five positive equilibrium points, in each case We proved that the general dynamical behavior is globally stable, except for the trivial equilibrium point (which was a saddle point)

The dynamical behavior in the case of good situations presented that the persistence dynamics is only a stable dynamical behavior

Through numerical simulations, we presented effects of stochastic fluctuations on interactions, which showed that noise strength led to an increase in the oscillations in dynamical behavior and became more complex, finally leading to extinction when the noise strength was high.

\section{Data Availability}

The data used to support the findings of this study are included within the article.

\section{Conflicts of Interest}

The authors declare that they have no conflicts of interest regarding the publication of this paper.

\section{Acknowledgments}

The authors extend their appreciation to the Deanship of Scientific Research at Majmaah University for funding this work under project no. R.G.P. 2019-4.

\section{References}

[1] J. Maynard-Smith, Models in Ecology, Cambridge University Press, London, UK, 1978.

[2] R. Haberman, Mathematical Models Mechanical Vibrations, Population Dynamics, and Traffic Flow, Society forIndustrial and Applied Mathematics, Philadelphia, PA, USA, 1998. 
[3] J. D. Murray, Mathematical Biology, vol. 2, Springer, New York, NY, USA, 2002.

[4] L. L. Rockwood, Introduction to Population Ecology, Blackwell Publishing, Malden, MA, USA, 2006.

[5] J. Pastor, Mathematical Ecology of Populations and Ecosystems, Wiley-Blackwell, Hoboken, NJ, USA, 2008.

[6] R. K. Upadhyay, R. K. Naji, and N. Kumari, "Dynamical complexity in some ecological models: effects of toxin production by phytoplankton," Nonlinear Analysis: Modelling and Control, vol. 12, no. 1, pp. 123-138, 2007.

[7] A. J. Lotka, Elements of Physical Biology, Williams \& Wilkins Company, Philadelphia, PA, USA, 1925.

[8] V. Volterra, "Variazioni e fluttuazioni del numerod'individui in specie animaliconviventi," Memoria della Reale Accademia Nazionale dei Lincei, vol. 2, pp. 31-113, 1926.

[9] K. Manna, V. Volpert, and M. Banerjee, "Dynamics of a diffusive two-prey-one-predator model with nonlocal intraspecific competition for both the prey specie," Mathematics, vol. 8, no. 101, 2020.

[10] U. E. Kocamaz, A. Göksu, H. Taşkın et al., "Control of chaotic two-predator one-prey model with single state control signals," Journal of Intelligent Manufacturing, 2020.

[11] J. Wu, "Dynamics of a two-predator one-prey stochastic delay model with Lévy noise," Physica A: Statistical Mechanics and its Applications, Elsevier, vol. 539, no. C, 2020.

[12] S. Gakkhar and R. K. Naji, "Seasonally perturbed preypredator system with predator-dependent functional response," Chaos, Solitons \& Fractals, vol. 18, no. 5, pp. 1075-1083, 2003.

[13] S. Zhang, D. Tan, and L. Chen, "Chaos in periodically forced Holling type II predator-prey system with impulsive perturbationsts," Chaos, Solitons \& Fractals, vol. 28, no. 2, pp. 367-376, 2006.

[14] R. K. Upadhyay and S. Iyengar, "Effect of seasonality on the dynamics of 2 and 3 species prey-predator systems," Nonlinear Analysis: Real World Applications, vol. 6, no. 3, pp. 509-530, 2007.

[15] H. Yu, S. Zhong, R. P. Agarwal, and S. K. Sen, "Effect of seasonality on the dynamical behavior of an ecological system with impulsive control strategy," Journal of the Franklin Institute, vol. 348, no. 4, pp. 652-670, 2011.

[16] J. Alebraheem, "Fluctuations in interactions of prey predator systems," Science International, vol. 28, pp. 2357-2362, 2016.

[17] X. Wang, H. Wang, and M. Y. Li, "R0 and sensitivity analysis of a predator-prey model with seasonality and maturation delay," Mathematical Biosciences, vol. 315, Article ID 108225, 2019.

[18] M. N. Hassan, L. Asik, J. Kulik, K. R. Long, and A. Peace, "Environmental seasonality on predator-prey systems under nutrient and toxicant constraints," Journal of Theoretical Biology, vol. 480, pp. 71-80, 2019.

[19] R. M. May, Stability and Complexity in Model Ecosystems, Princeton University Press, Princeton, NJ, USA, 1974.

[20] G. P. Samanta, "Stochastic analysis of a prey-predator system," International Journal of Mathematical Education in Science and Technology, vol. 25, no. 6, pp. 797-803, 1994.

[21] M. Bandyopadhyay and C. G. Chakrabarti, "Deterministic and stochastic analysis of a nonlinear prey-predator system," Journal of Biological Systems, vol. 11, no. 2, pp. 161-172, 2003.

[22] G. Q. Cai and Y. K. Lin, "Stochastic analysis of the LotkaVolterra model for ecosystems," Physical Review E, vol. 70, Article ID 041910, 2004.

[23] A. Maiti and G. P. Samanta, "Deterministic and stochastic analysis of a ratio-dependent prey-predator system,"
International Journal of Systems Science, vol. 37, no. 12, pp. 817-826, 2006.

[24] L. M. Addison, "Analysis of a predator-prey model: a deterministic and stochastic approach," Journal of Biometrics \& Biostatistics, vol. 8, no. 4, 2017.

[25] J. Alebraheem, "Paradox of enrichment in a stochastic predator-prey model," Journal of Mathematics, vol. 2020, Article ID 8864999, 8 pages, 2020.

[26] Y. Kuang and E. Beretta, "Global qualitative analysis of a ratiodependent predator-prey system," Journal of Mathematical Biology, vol. 36, no. 4, pp. 389-406, 1998.

[27] H. I. Freedman and P. Waltman, "Persistence in models of three interacting predator-prey populations," Mathematical Biosciences, vol. 68, no. 2, pp. 213-231, 1984.

[28] B. Dubey and R. K. Upadhyay, "Persistence and extinction of one-prey and two-predators system," Nonlinear Analysis: Modelling and Control, vol. 9, no. 4, pp. 307-329, 2004.

[29] J. Alebraheem and Y. A. Hasan, "Dynamics of a two predatorone prey system," Computational and Applied Mathematics, vol. 33, no. 3, pp. 767-780, 2014.

[30] J. Alebraheem, "Relationship between the paradox of enrichment and the dynamics of persistence and extinction in prey-predator systems," Symmetry, vol. 10, no. 10, p. 532, 2018.

[31] M. J. Keeling, P. Rohani, and B. T. Grenfell, "Seasonally forced disease dynamics explored as switching between attractors," Physica D: Nonlinear Phenomena, vol. 148, no. 3-4, pp. 317335, 2001.

[32] R. Olinky, A. Huppert, and L. Stone, "Seasonal dynamics and thresholds governing recurrent epidemics," Journal of Mathematical Biology, vol. 56, no. 6, pp. 827-839, 2008. 